\title{
Objectively Measured Sleep Characteristics and Incidence of Ischemic Stroke: The Sleep Heart Health Study
}

\author{
Binbin Zhaol,* \\ Yanhua $\mathrm{Wu}^{2}, *$ \\ Xiaoying Jin' \\ Lihong Yang ${ }^{2}$ \\ Jian Yang 1,2 \\ Xiancang $\mathrm{Ma}^{\prime}$ \\ Bin Yan $\mathbb{I D}^{1,2}$
}

'Department of Psychiatry, The First Affiliated Hospital of Xi'an Jiaotong University, Xi'an, People's Republic of China; ${ }^{2}$ Department of Clinical Research Center, The First Affiliated Hospital of Xi'an Jiaotong University, Xi'an, People's Republic of China

*These authors contributed equally to this work
Correspondence: Bin Yan

Department of Clinical Research Center,

The First Affiliated Hospital of Xi'an

Jiaotong University, Xi'an, People's

Republic of China

Tel +86 29853236/4

Email yanbin.1987@hotmail.com
Objective: Sleep disorders are associated with the prevalence of stroke. However, there is limited evidence regarding the association between objectively measured sleep characteristics and ischemic stroke.

Methods: Ischemic stroke was assessed during the mean follow-up period of 11 years in the Sleep Heart Health Study. Sleep parameters such as wake after sleep onset (WASO) and sleep efficiency (SE) were objectively measured based on in-home polysomnography records. Multivariable Cox regression analysis was utilized to examine the relationship between objective sleep characteristics and ischemic stroke incidence.

Results: This study involved 4204 participants (1978 males and 2226 females, 63.8 \pm 11.1 years). The incidence of ischemic stroke increased in individuals with long WASO, poor SE, and short sleep duration. Multivariable Cox regression analysis showed that WASO within the fourth quartile (hazard ratio [HR] 3.771, 95\% confidence interval [CI] 1.805-7.877, $\mathrm{P}<0.001$ ), third quartile (HR 3.009, 95\% CI 1.433-6.317, $\mathrm{P}=0.004$ ), and second quartile (HR $3.108,95 \%$ CI $1.470-6.568, \mathrm{P}=0.003$ ) had a higher incidence of ischemic stroke than WASO within the first quartile. Poor SE $(<80.0 \%)$ was also found to be a predictor for ischemic stroke (HR 2.220, 95\% CI 1.244-3.960, $\mathrm{P}=0.007$ ). Additionally, a short sleep duration $(<6 \mathrm{~h})$ was associated with an increased risk of ischemic stroke (HR 1.725, 95\% CI 1.026-2.899, $\mathrm{P}=0.040$ ).

Conclusion: Our results revealed a relationship between WASO, SE, and sleep duration and ischemic stroke. Therefore, these sleep characteristics may be adequate predictors for the incidence of ischemic stroke.

Keywords: sleep characteristics, ischemic stroke, polysomnography, Sleep Heart Health Study

\section{Introduction}

Stroke is one of the most common causes of severe disability and death in the world. ${ }^{1}$ The stroke burden is expected to rise from approximately 38 million disability-adjusted life-years globally in 1990 to 61 million disability-adjusted lifeyears in $2020 .^{2}$ Ischemic stroke is the most prevalent type of stroke, accounting for nearly $88 \%$ of all types. ${ }^{3}$ There are many common risk factors for ischemic stroke, including aging, smoking, tobacco use, and high blood pressure. ${ }^{4-6}$ Moreover, studies have revealed that sleep habits such as daytime napping and caloric intake before bedtime increase the risk of ischemic stroke and the prevalence of its risk factors. $^{7-10}$ 
A growing number of studies investigated the role of objective sleep characteristics on cardiovascular disease and its risk factors based on polysomnography (PSG) and actigraphy. ${ }^{11-13}$ Objective sleep characteristics such as sleep efficiency (SE), arousal index (ArI), the sleep fragmentation index, the percentage of rapid eye movement (REM sleep \%), and the percentage of slow-wave sleep (SWS \%) were found to be associated with cardiovascular disease and related risk factors, including hypertension, diabetes mellitus, and metabolic syndrome. ${ }^{13-17}$ Von Kanel et al also showed that long wake after sleep onset (WASO) was associated with an increased risk of coronary artery disease. ${ }^{18}$ However, there is limited evidence regarding the association between objectively measured sleep characteristics and ischemic stroke based on PSG records.

In this study, we aimed to explore the role of objective sleep characteristics, including SE, WASO, sleep architecture (REM sleep \% and SWS\%), sleep fragmentation (SFI and ArI), sleep latency, and sleep duration, on the incidence of ischemic stroke based on a large communitybased population from the database of the Sleep Heart Health Study (SHHS).

\section{Materials and Methods}

\section{Study Population}

The SHHS is a community-based multicenter prospective cohort study that investigated the incidence of cardiovascular disease and the consequences of sleep-disordered breathing (ClinicalTrials.gov Identifier: NCT00005275). The design and quality control procedures have been previously reported. ${ }^{19}$ The study population was selected from multicenter prospective cohort studies, including the Framingham Offspring and Omni Study, the Strong Heart Study, the Cardiovascular Health Study, the Atherosclerosis Risk in Communities Study, and the cohort studies of respiratory diseases in Tucson. All the participants provided written informed consent, and the study was approved by the Institutional Review Board of each participating institution, including Boston University, Case Western Reserve University, Johns Hopkins University, Missouri Breaks Research Inc., New York University Medical Center, University of Arizona, University of California at Davis, University of Minnesota-Clinical and Translational Science Institute, and the University of Washington. We had access to the SHHS database through a signed agreement with Brigham and Women's Hospital.

\section{Inclusion and Exclusion Criteria}

We obtained data on 5804 participants from SHHS datasets. We included individuals with complete PSG records. Participants were excluded in the present study if they (1) had a history of stroke, (2) had no follow-up data, and (3) reported that their sleep during the nighttime was affected by PSG and was significantly different than usual (based on responses to questions such as "Compared to your usual night's sleep, how well did you sleep last night?" collected in the morning survey questionnaires) (Figure 1). Finally, a total of 4204 participants were included in the present study.

\section{Sleep Characteristics}

All participants in the current investigation underwent overnight electroencephalography-based PSG (P-Series; Compumedics, Abbotsville, Australia) in their homes by trained and certified technicians. Details regarding the specific technical aspects of the PSG measurement are shown in the Supplement Methods. WASO is defined as the total amount of arousal time between the beginning of sleep and the ultimate wake time. In the present study,

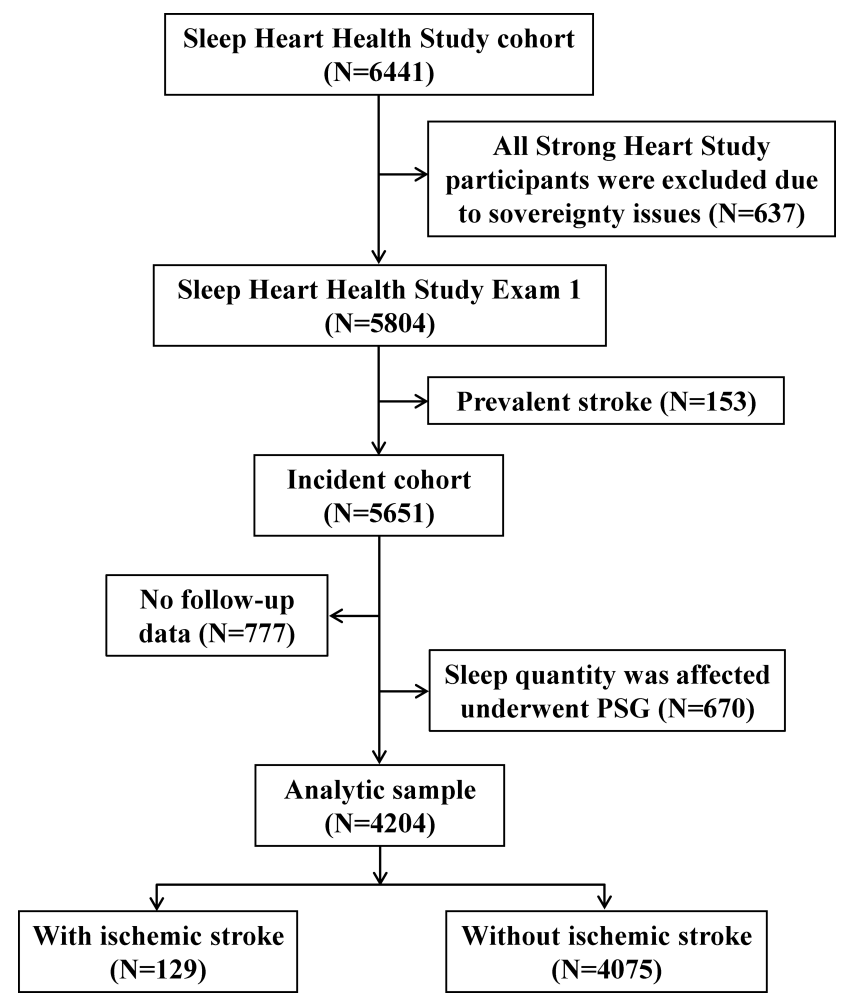

Figure I Inclusion and exclusion criteria for the study population. This figure depicts the inclusion of patients into the current investigation as a flowchart. The arrows that branch off represent patients that did not meet the criteria and were excluded. 
WASO was also categorized into a first quartile $(<30.0$ $\mathrm{min}$ ), second quartile (30.0 $\mathrm{min}-48.5 \mathrm{~min}$ ), third quartile (49.0 min-80 $\mathrm{min})$, and fourth quartile (>80 $\mathrm{min})$. SE, defined as the ratio of time spent asleep to the total time in bed multiplied by 100 , was divided into a first quartile $(<80 \%)$, second quartile $(80-84.9 \%)$, third quartile ( $85-$ $89.9 \%$ ), and fourth quartile ( $\geq 90 \%)$. Sleep duration in the present study was defined as the total time in bed, calculated as the time between lights off and lights on (rounded to the nearest minute). In addition, sleep duration was also split into three groups, including short sleep duration $(<6$ $\mathrm{h}$ per night), normal sleep duration (6-8 h per night), and long sleep duration ( $>8 \mathrm{~h}$ per night). Sleep latency was determined as the time from lights off to sleeping. ArI was calculated as the ratio of number of arousals to the total sleep time. REM sleep \% was defined as the percentage of REM sleep captured by PSG. Slow-wave sleep was presented as the third stage of non-REM sleep. The sleep fragmentation index is determined by dividing the total number of awakenings and sleep stage shifts by the total sleep time multiplied by 100 . Apnea is defined as a complete or almost complete cessation of airflow, lasting at least 10 seconds, and is usually associated with desaturation or an arousal. Hypopnea is a reduction in airflow ( $<70 \%$ of a "baseline" level), associated with desaturation or arousal. The apnea-hypopnea index (AHI) was calculated as all apnea and hypopnea episodes per hour of sleep accompanied by at least a $4 \%$ drop in oxygen saturation. Sleep disorder breathing is a condition characterized by repeated episodes of hypopnea and apnea, defined as AHI $\geq 5$ events/h in the present study.

\section{Outcomes}

The incidence of ischemic stroke was evaluated according to the patient cohorts based on exhaustive protocols. ${ }^{19}$ Ischemic stroke was defined as the first occurrence between the baseline PSG and the end of follow-up. The medical records were extracted when potential ischemic stroke was identified. Ischemic stroke was further investigated and confirmed by symptoms, physical findings, the presence of a non-carotid embolic source, the results of computed tomography and magnetic resonance imaging examinations, cerebral angiograms and lumbar punctures, and pathology reports.

\section{Covariates}

The participants' age, gender, body mass index (BMI), race, smoking status, history of diabetes mellitus and hypertension, benzodiazepine use, alcohol use, and PSG data were obtained from the baseline examination of the SHHS.

\section{Statistical Analysis}

Analysis of variance (ANOVA) was performed to compare differences between continuous variables, while the chi-square test was used for categorical variables. Continuous variables were expressed as mean \pm standard deviation, and categorical variables were identified as percentages. Unadjusted Kaplan-Meier plots were used to evaluate the association of WASO, SE, and sleep duration with ischemic stroke. Univariable and multivariable Cox regression analyses were performed to estimate the association between each individual's sleep characteristics and the incidence of ischemic stroke. Covariates, including age, gender, smoking status, race, BMI, prevalent hypertension and diabetes mellitus, apnea-hypopnea index, benzodiazepine use, alcohol use, and sleep duration, were included in the final Cox regression model. The hazard ratio (HR) and 95\% confidence intervals (CI) were utilized to examine the relationship between objectively measured sleep characteristics and the incidence of ischemic stroke. The analysis of interaction terms was also constructed between objective sleep characteristics and other covariates on the incidence of ischemic stroke. All statistical analyses were performed using Statistical Package for Social Sciences (SPSS) statistics software (v. 24.0, SPSS Inc., Chicago, IL), and $P<0.05$ was considered statistically significant.

\section{Results}

\section{Participant Characteristics}

In the present study, 4204 participants (1978 males and 2226 females, $63.8 \pm 11.1$ years) were enrolled. During the mean follow-up period of $10.7 \pm 3.0$ years, 129 cases of ischemic stroke were observed. Participants with long WASO were older and more likely to be male. Participants within the fourth quartile of WASO had the highest proportion of smokers, diabetes mellitus, and hypertension compared to the third, second, and first quartiles. In addition, long WASO was associated with a high level of AHI (Table 1). Supplement Table 1 also provides the sleep characteristics in participants with $(n=129)$ or without ischemic stroke $(n=4075)$. Individuals with ischemic stroke were prone to short 
Table I Descriptive Characteristics by WASO Quartile (Quartile I: <30.0min; Quartile II: 30.0min-48.5min; Quartile III:49.0min80.0min; Quartile IV: >80.0min)

\begin{tabular}{|c|c|c|c|c|c|c|}
\hline \multirow[t]{2}{*}{ Characteristics } & \multirow{2}{*}{$\begin{array}{c}\text { Total } \\
(n=4204)\end{array}$} & \multicolumn{4}{|c|}{ WASO (Min) } & \multirow[b]{2}{*}{$P$ value } \\
\hline & & $\begin{array}{l}\text { First Quartile } \\
\qquad(n=1048)\end{array}$ & $\begin{array}{l}\text { Second Quartile } \\
\qquad(n=1063)\end{array}$ & $\begin{array}{l}\text { Third Quartile } \\
\qquad(n=1047)\end{array}$ & $\begin{array}{l}\text { Fourth Quartile } \\
\qquad(n=1046)\end{array}$ & \\
\hline Age, year & $63.8 \pm 11.1$ & $59.2 \pm 11.0$ & $61.7 \pm 10.6$ & $65.5 \pm 10.3$ & $68.7 \pm 10.2$ & $<0.001$ \\
\hline Gender, n (\%) & & & & & & $<0.001$ \\
\hline Male & $1978(47.1)$ & $405(38.6)$ & $470(44.2)$ & $537(5 \mathrm{I} .3)$ & $566(54.1)$ & - \\
\hline Female & $2226(52.9)$ & $643(6 I .4)$ & $593(55.8)$ & $510(48.7)$ & $480(45.9)$ & - \\
\hline Body mass index, $\mathrm{kg} / \mathrm{m}^{2}$ & $28.2 \pm 5.0$ & $28.2 \pm 5.2$ & $28.0 \pm 4.9$ & $28.4 \pm 4.8$ & $28.4 \pm 5.0$ & 0.122 \\
\hline Race, n (\%) & & & & & & 0.076 \\
\hline White & $3668(87.3)$ & $907(86.5)$ & $919(86.5)$ & $938(89.6)$ & $904(86.4)$ & - \\
\hline Other & $536(12.7)$ & $14 \mid(\mid 3.5)$ & $144(13.5)$ & $109(10.4)$ & $142(13.6)$ & - \\
\hline Smoking status, n (\%) & & & & & & 0.044 \\
\hline Smoker & $2260(53.8)$ & $546(52.1)$ & $549(5 \mid .7)$ & $568(54.3)$ & $597(57.2)$ & - \\
\hline Never smoker & 1938 (46.2) & $502(47.9)$ & $512(48.3)$ & $478(45.7)$ & $446(42.8)$ & - \\
\hline Alcohol use, n (\%) & & & & & & 0.170 \\
\hline At least I drink per day & I $708(43.3)$ & $419(42.9)$ & $457(45.9)$ & $399(4 I .0)$ & $433(43.3)$ & - \\
\hline None & $2238(56.7)$ & $557(57.1)$ & $538(54.1)$ & $575(59.0)$ & $568(56.7)$ & - \\
\hline Benzodiazepine use, n (\%) & $246(5.9)$ & $48(4.6)$ & $65(6.1)$ & $59(5.6)$ & $74(7.1)$ & 0.105 \\
\hline Diabetes mellitus, n (\%) & $295(7.0)$ & $55(5.2)$ & $53(5.0)$ & $93(8.9)$ & $94(9.0)$ & $<0.001$ \\
\hline Hypertension, n (\%) & $|68|(40.0)$ & $309(29.5)$ & $382(35.9)$ & $447(42.7)$ & $543(51.9)$ & $<0.001$ \\
\hline$A H I$, events/h & $9.9 \pm 13.0$ & $7.8 \pm 11.2$ & $8.9 \pm 12.2$ & $10.3 \pm 12.5$ & $12.5 \pm 15.4$ & $<0.001$ \\
\hline Sleep duration, $\mathrm{h}$ & $7.3 \pm 0.9$ & $7.0 \pm 1.0$ & $7.2 \pm 0.9$ & $7.4 \pm 0.9$ & $7.7 \pm 0.8$ & $<0.001$ \\
\hline $\mathrm{SE}, \%$ & $83.4 \pm 10.0$ & $91.6 \pm 5.5$ & $88.0 \pm 4.5$ & $82.7 \pm 4.9$ & $71.1 \pm 9.5$ & $<0.001$ \\
\hline REM, \% & $19.7 \pm 6.8$ & $21.3 \pm 6.6$ & $20.3 \pm 6.3$ & $19.3 \pm 6.7$ & $17.8 \pm 7.1$ & $<0.001$ \\
\hline SWS, \% & $17.5 \pm 11.9$ & $19.9 \pm 12.1$ & $18.3 \pm 1 \mid .4$ & $15.9 \pm 1 \mid .5$ & $16.0 \pm 12.2$ & $<0.001$ \\
\hline $\mathrm{SL}, \min$ & $12.8 \pm 19.5$ & $14.0 \pm 2 \mid .5$ & $12.4 \pm 18.6$ & $12.7 \pm 18.5$ & $12.2 \pm 19.2$ & 0.177 \\
\hline Total Arl, events/h & $19.0 \pm 10.4$ & $15.4 \pm 8.6$ & $18.0 \pm 8.8$ & $20.1 \pm 10.0$ & $22.7 \pm 12.3$ & $<0.001$ \\
\hline Arl-REM, events/h & $15.2 \pm 10.8$ & $13.7 \pm 10.0$ & $15.0 \pm 10.6$ & $15.3 \pm 10.2$ & $16.7 \pm 12.3$ & $<0.001$ \\
\hline Arl-NREM, events/h & $19.9 \pm 11.3$ & $15.8 \pm 9.3$ & $18.7 \pm 9.5$ & $21.2 \pm 11.2$ & $24.0 \pm 13.3$ & $<0.001$ \\
\hline SFI, events/h & $8.9 \pm 3.4$ & $7.4 \pm 2.6$ & $8.5 \pm 2.8$ & $9.0 \pm 3.0$ & $10.5 \pm 4.2$ & $<0.001$ \\
\hline Follow-up time, year & $10.7 \pm 3.0$ & $11.2 \pm 2.7$ & $11.0 \pm 2.8$ & $10.7 \pm 2.9$ & $10.0 \pm 3.4$ & $<0.001$ \\
\hline
\end{tabular}

Notes: Results are presented as mean \pm standard deviation or $\mathrm{n}(\%)$. The $\mathrm{P}$ values represent the difference among four groups.

Abbreviations: AHI, apnea hypopnea index; Arl, arousal index; NREM, non-rapid eye movement; REM, rapid eye movement; SFI, sleep fragmentation index; SL, sleep latency; SWS, slow wave sleep; WASO, wake after sleep onset.

sleep durations, long WASO, poor SE, and high levels of AHI. Baseline characteristics data classified by sleep efficiency and sleep duration are provided in Supplement Tables 2 and 3.

\section{Ischemic Stroke and WASO, SE, and Sleep} Duration

There were significant differences in the distribution of incident ischemic stroke in the fourth WASO quartile 
A

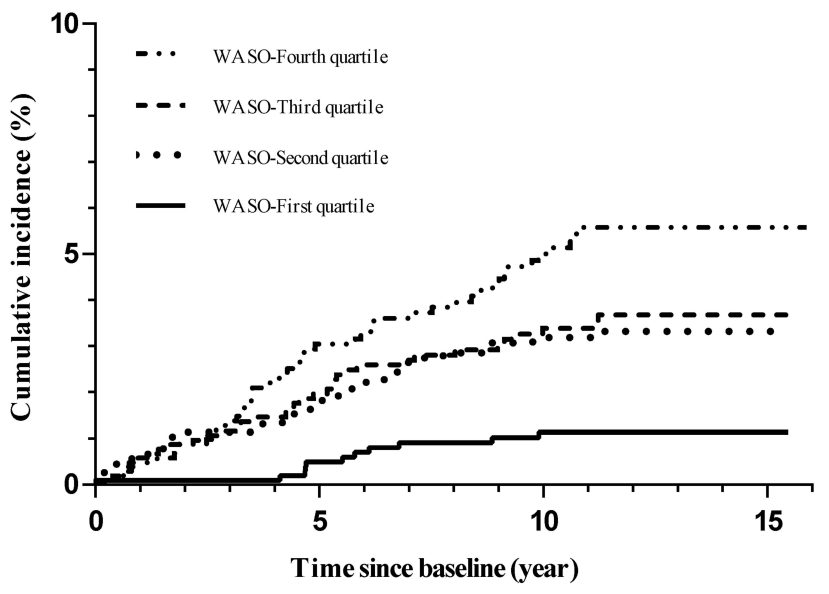

B

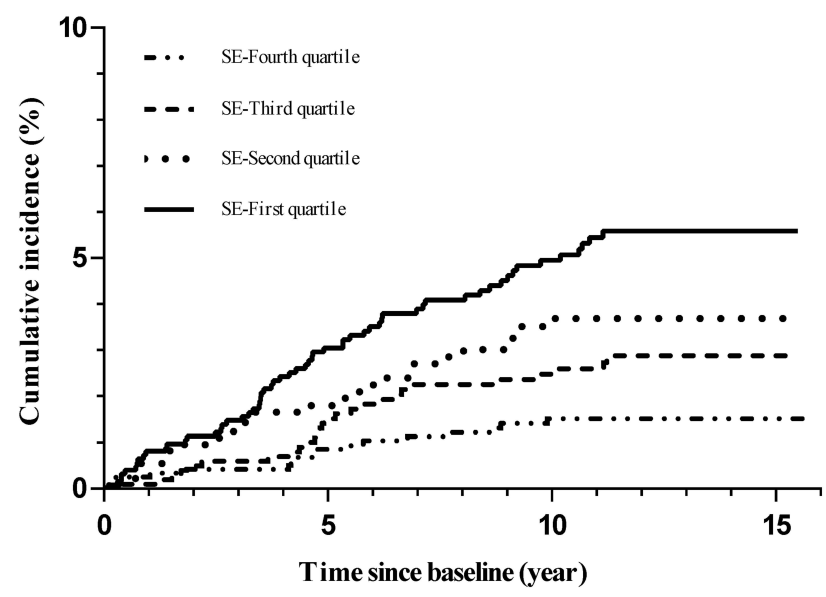

Figure 2 Kaplan-Meier plots of cumulative risk for ischemic stroke stratified by WASO quartiles ((A); quartile I $<30.0$ min; quartile II: 30.0 min-48.5 min; quartile III: 49.0 min-80.0 min; quartile IV: $>80.0 \mathrm{~min}$ ) and SE ((B); quartile I <80\%, quartile II: $80 \%-84.9 \%$, quartile III: $85 \%-89.9 \%$, and quartile IV: $\geq 90 \%)$.

$(\mathrm{P}<0.001)$. Unadjusted Kaplan-Meier analysis showed an increased incidence of ischemic stroke among participants who had long WASO (Figure 2A). After adjusting for age, gender, smoking status, race, BMI, hypertension, diabetes mellitus, AHI, benzodiazepine use, alcohol use, and sleep duration, WASO (per min) (HR 1.004, 95\% CI 1.000$1.008, \mathrm{P}=0.028$ ) was found to be a predictor for the incidence of ischemic stroke (Table 2). HRs for WASO (continuous) associated with ischemic stroke were relatively small. We further explored the role of WASO classified by quartiles on the incidence of ischemic stroke. The results showed that the WASO of the fourth quartile (HR 3.771, 95\% CI 1.805-7.877, $\mathrm{P}=0.001$ ), third quartile (HR $3.009,95 \%$ CI $1.433-6.317, \mathrm{P}=0.004)$, and second quartile

Table 2 HRs and 95\% Cls for Sleep Characteristics Associated with Ischemic Stroke

\begin{tabular}{|c|c|c|c|c|c|c|}
\hline & Univariable Models & & Multivariable Adjusted ${ }^{\dagger}$ & & Multivariable Adjusted ${ }^{\ddagger}$ & \\
\hline Sleep Variables & HR (95\% Cl) & $\mathbf{P}$ & HR $(95 \% \mathrm{Cl})$ & $\mathbf{P}$ & HR $(95 \% \mathrm{Cl})$ & $\mathbf{P}$ \\
\hline WASO & $1.008(1.005-1.011)$ & $<0.001$ & $1.003(1.000-1.007)$ & 0.049 & $1.004(1.000-1.008)$ & 0.028 \\
\hline SE & $0.964(0.951-0.977)$ & $<0.001$ & $0.980(0.966-0.995)$ & 0.011 & $0.981(0.966-0.996)$ & 0.012 \\
\hline REM, \% & $0.97 \mid(0.947-0.995)$ & 0.017 & $0.993(0.969-1.019)$ & 0.598 & $0.994(0.970-1.020)$ & 0.665 \\
\hline SWS, \% & $0.993(0.978-1.008)$ & 0.361 & $0.992(0.977-1.008)$ & 0.346 & $0.992(0.976-1.007)$ & 0.300 \\
\hline SL & $1.003(0.994-1.011)$ & 0.551 & $1.002(0.993-1.011)$ & 0.639 & $1.002(0.993-1.010)$ & 0.693 \\
\hline SFI & $1.036(0.987-1.087)$ & 0.149 & I.007 (0.959-I.057) & 0.780 & $1.006(0.958-1.056)$ & 0.815 \\
\hline Total Arl & $1.002(0.985-1.019)$ & 0.798 & $0.992(0.972-1.013)$ & 0.462 & $0.992(0.972-1.013)$ & 0.450 \\
\hline Arl-REM & $0.986(0.968-1.004)$ & 0.128 & $0.986(0.967-1.005)$ & 0.149 & $0.987(0.968-1.005)$ & 0.162 \\
\hline Arl-NREM & $1.005(0.990-1.020)$ & 0.539 & $0.995(0.978-1.014)$ & 0.623 & $0.995(0.977-1.013)$ & 0.607 \\
\hline \multicolumn{7}{|l|}{ Sleep duration } \\
\hline$<6 \mathrm{~h}$ & $1.943(1.173-3.220)$ & 0.010 & $1.725(1.026-2.899)$ & 0.040 & & \\
\hline$>8 \mathrm{~h}$ & $1.326(0.893-1.970)$ & 0.162 & $1.223(0.816-1.833)$ & 0.330 & & \\
\hline $6 h-8 h$ & I (Ref) & & I (Ref) & & & \\
\hline
\end{tabular}

Notes: ${ }^{\dagger}$ Each individual sleep characteristics was adjusted by age, gender, smoking status, race, body mass index, prevalent hypertension and diabetes mellitus, apneahypopnea index, benzodiazepine use, alcohol use. ${ }^{\ddagger}$ Adjusted by ${ }^{\dagger}$ plus sleep duration.

Abbreviations: Arl, arousal index; $95 \% \mathrm{Cl}$, $95 \%$ confidence interval; HR, hazard ratio; NREM, non-rapid eye movement; REM, rapid eye movement; SFI, sleep fragmentation index; SL, sleep latency; SWS, slow wave sleep. 
Table 3 HRs and 95\% Cls for Quartiles of WASO and SE Associated with Ischemic Stroke

\begin{tabular}{|c|c|c|c|c|c|c|}
\hline \multirow[b]{2}{*}{ All Subjects } & Univariable Models & \multirow[b]{2}{*}{$\mathbf{P}$} & \multirow{2}{*}{$\begin{array}{c}\text { Multivariable Adjusted }{ }^{\dagger} \\
\text { HR }(95 \% \mathrm{Cl})\end{array}$} & \multirow[b]{2}{*}{$\mathbf{P}$} & \multirow{2}{*}{$\begin{array}{c}\text { Multivariable Adjusted }{ }^{\ddagger} \\
\text { HR }(95 \% \mathrm{Cl})\end{array}$} & \multirow[b]{2}{*}{$\mathbf{P}$} \\
\hline & HR $(95 \% \mathrm{Cl})$ & & & & & \\
\hline \multicolumn{7}{|l|}{ WASO } \\
\hline Fourth quartile (>80 min) & $4.996(2.600-9.597)$ & $<0.001$ & $3.298(1.608-6.768)$ & 0.001 & $3.77 \mid(1.805-7.877)$ & $<0.001$ \\
\hline Third quartile (49.0min-80min) & $3.299(1.675-6.496)$ & 0.001 & $2.749(1.317-5.738)$ & 0.007 & $3.009(1.433-6.317)$ & 0.004 \\
\hline Second quartile (30.0min-48.5min) & $3.005(1.519-5.945)$ & 0.002 & $2.933(1.392-6.183)$ & 0.005 & $3.108(1.470-6.568)$ & 0.003 \\
\hline First quartile $(<30.0 \mathrm{~min})$ & $\mathrm{I}(\operatorname{Ref})$ & & $\mathrm{I}(\operatorname{Ref})$ & & I (Ref) & \\
\hline \multicolumn{7}{|l|}{ Sleep efficiency } \\
\hline First quartile $(<80.0 \%)$ & $3.726(2.174-6.386)$ & $<0.001$ & $2.210(1.241-3.936)$ & 0.007 & $2.220(1.244-3.960)$ & 0.007 \\
\hline Second quartile (80.0-84.9\%) & $2.467(1.332-4.569)$ & 0.004 & $1.859(0.970-3.564)$ & 0.062 & $1.829(0.952-3.514)$ & 0.070 \\
\hline Third quartile (85.0-89.9\%) & $1.866(1.017-3.423)$ & 0.044 & $1.443(0.762-2.732)$ & 0.261 & $1.457(0.769-2.760)$ & 0.248 \\
\hline Fourth quartile ( $(\geq 90 \%)$ & I(Ref) & & I (Ref) & & I (Ref) & \\
\hline
\end{tabular}

Notes: ${ }^{\dagger}$ Adjusted by age, gender, smoking status, race, body mass index, prevalent hypertension and diabetes mellitus, apnea-hypopnea index, benzodiazepine use, alcohol use. ${ }^{\ddagger}$ Adjusted by ${ }^{\dagger}$ plus sleep duration.

Abbreviations: $95 \% \mathrm{Cl}, 95 \%$ confidence interval; HR, hazard ratio; WASO, wake after sleep onset.

(HR 3.108, 95\% CI 1.470-6.568, $\mathrm{P}=0.003$ ) increased the risk of ischemic stroke incidence compared with that of the first quartile (Table 3).

Moreover, the occurrence of ischemic stroke increased in individuals with poor SE according to the unadjusted Kaplan-Meier analysis (Figure 2B). Multivariable Cox regression analysis showed that $\mathrm{SE}$ (per $1 \%$ increase) (HR $0.981,95 \%$ CI $0.966-0.996, \mathrm{P}=0.012$ ) was associated with a reduced risk of ischemic stroke (Table 2). Moreover, compared with $\mathrm{SE} \geq 90 \%$, poor SE $(<80.0 \%)$ increased the risk of ischemic stroke (HR 2.220, 95\% CI 1.244-3.960, $\mathrm{P}=0.007$ ) (Table 3). Furthermore, a short sleep duration $(<6$ h) was also a risk factor for the incidence of ischemic stroke (HR 1.725, 95\% CI 1.026-1.833, $\mathrm{P}=0.040$ ).

\section{Ischemic Stroke and Other Sleep Characteristics}

We also investigated the association between ischemic stroke and REM sleep \%, SWS \%, sleep latency, sleep fragmentation index, and ArI. However, no significant difference was found in the multivariable Cox regression analysis (Table 2). An analysis of the association between $\mathrm{AHI}$ and ischemic stroke was also performed. AHI levels of 5.0-14.9 events/hour are associated with a higher risk of ischemic stroke compared with controls (AHI $<5$ events/hour) (Supplement Table 4). No significant association of AHI levels $\geq 30.0$ events/hour and 15.0-29.9 events/hour with ischemic stroke was found.

\section{Interaction Analysis}

We further investigated the possible interaction effects between sleep characteristics and sleep disorder breathing
( $<15$ events/hour vs $\geq 15$ events/hour) on the incidence of ischemic stroke. No significant interaction was found (Supplement Table 5). We also investigated the association of sleep characteristics with the incidence of ischemic stroke stratified by gender. The results showed that WASO, SE, and short sleep duration were associated with the female but male sex. However, no significant interaction was found in these analyses (Supplement Table 6).

\section{Discussion}

Increasing evidence shows that sleep disorders are closely related to stroke. ${ }^{7,8}$ In the present study, we investigated the association between ischemic stroke and objectively measured sleep characteristics based on PSG in a middleage- and older community-based population. Our findings demonstrated that long WASO, poor SE, and short sleep duration were associated with an increased risk of ischemic stroke in middle aged and older adults.

PSG and wrist actigraphy are important ways to measure objective sleep characteristics. ${ }^{18,20,21}$ Sleep parameters such as WASO and SE could be obtained from PSG records. WASO is an important sleep parameter measured by the periods of wakefulness after defined sleep onset. ${ }^{22}$ Von Kanel et al demonstrated that WASO was a predictor for morning fasting plasma levels of von Willebrand Factor antigen and soluble tissue factor antigen, which are closely related to the risk of coronary artery disease. ${ }^{18}$ SE refers to the percentage of total time in bed actually spent in sleep, which could reflect the general estimation of the overall quality of sleep. ${ }^{22}$ Studies showed that SE was associated with risk factors of ischemic stroke, including BMI, hypertension, and 
insulin sensitivity in adults. ${ }^{15}$ These studies suggested that WASO and SE are closely related to ischemic stroke risk factors. However, little is known about the role of WASO and SE in the occurrence of ischemic stroke. In our analysis of SHHS data, individuals with long WASO and poor SE had an increased risk of ischemic stroke incidence. To provide more useful information about the role of WASO and SE on the incidence of ischemic stroke, we classified WASO and SE by quartiles. Our findings showed that WASO $>80 \mathrm{~min}$ and $\mathrm{SE}<80 \%$ significantly contributes to the incidence of ischemic stroke. Our findings indicate that WASO and SE based on PSG records may be predictors for the incidence of ischemic stroke.

Many studies have shown that sleep duration is closely related to quality of life and physical health. Sleep duration may be influenced by age, race, work status, education level, lifestyles, and environmental and medical conditions. $^{23,24}$ Sleep duration usually obtained from sleep questionnaires could also be measured by PSG and actigraphy. Studies showed that under- and over-reporting of sleep is common in middle-age and older population when compared with objectively measured sleep duration. ${ }^{25-28}$ Subjective reports of habitual sleep are correlated with objective measured sleep, but the true association between self-reported sleep duration and human health may be different. ${ }^{29,30}$ Many studies have explored the relationship between self-reported sleep duration and stroke. A systematic review showed that a self-reported sleep duration $>9 \mathrm{~h}$ or even $>10 \mathrm{~h}$ significantly increased the risk of ischemic stroke. ${ }^{31}$ Leng et al also demonstrated that a self-reported sleep duration $>8 \mathrm{~h}$ per night is related to a higher risk of stroke. ${ }^{32}$ A case-control study also showed that a self-reported sleep duration of $>8 \mathrm{~h}$ per night was associated with a high risk of ischemic stroke in Chinese populations. ${ }^{33}$ However, no significant association was found between a long sleep duration (defined as $>8 \mathrm{~h}$ per night) and ischemic stroke in our analysis. It may be caused by different definitions of long sleep duration or different study populations. In addition, Eguchi et al found that a self-reported sleep duration $<7.5 \mathrm{~h}$ was independently associated with the risk of ischemic stroke. ${ }^{34}$ Furthermore, a self-reported sleep duration $<6 \mathrm{~h}$ was also associated with ischemic stroke in postmenopausal women. ${ }^{35}$ In this study, we also found that a short sleep duration (defined as $<6 \mathrm{~h}$ per night) significantly increased the risk of ischemic stroke.
A previous study found that REM sleep $\%>25 \%$ was correlated with angina pectoris. ${ }^{16}$ Sleep fragmentation index and SWS \% were also demonstrated to be closely related to hypertension. ${ }^{15,17}$ Additionally, total ArI, ArIREM, and ArI-NREM were significantly associated with a high coronary artery calcification burden. ${ }^{36}$ The effects of REM sleep \%, SWS \%, sleep fragmentation index, total ArI, ArI-REM, ArI-NREM, and sleep latency on the incidence of ischemic stroke were also explored in this study. However, no significant relationship between these sleep characteristics and ischemic stroke was found in these analyses.

Sleep disorder breathing seriously affects the body's homeostasis and circadian rhythm, which can then cause various cardiovascular and cerebrovascular diseases. ${ }^{37}$ Redline et al showed that there was a significant association between sleep disorder breathing and ischemic stroke in middle-age and older men based on SHHS datasets. ${ }^{38}$ In the present study, participants with a high level of AHI had a higher incidence of ischemic stroke than did the controls. However, the associations were attenuated after multivariable adjustment. It may be due to the relatively small sample size of high level of AHI, especially in AHI levels $\geq 30.0$ events/hour and 15.0-29.9 events/hour. The participants with sleep disorder breathing may have microarousals during the night, which could affect SE and WASO. We therefore adjusted for AHI in our multivariable Cox regression analysis to explore the association between sleep characteristics and ischemic stroke. We also performed the subgroup analysis stratified by AHI ( $<15$ events/hour vs $\geq 15$ events/hour). No significant interaction was found between SE/WASO and AHI.

The potential mechanisms between objective sleep characteristics and ischemic stroke remain unclear. Poor sleep was correlated with cardiovascular risk factors such as hypertension, obesity, and type 2 diabetes. ${ }^{39}$ Individuals with poor SE and longer WASO were also more likely to have poor sleep quality, which could easily activate the sympathetic nervous system and lead to increased heart rate and blood pressure at nighttime. ${ }^{40} \mathrm{~A}$ recent study also found that mice with sleep fragmentation produce more Ly-6C monocytes, causing larger atherosclerotic lesions and the production of less hypocretin. ${ }^{41}$

Because SHHS data is based on a community population, our results are suitable for the general population. However, this study had a few limitations. Participants consisted of middle-age and older adults (63.8 \pm 11.1 years), and most were Caucasian (87.3\%). We observed 
that individuals with long WASO, low SE, and short sleep duration were more likely to be older. Therefore, our results should not be extended to all ethnic groups or young populations. Additionally, the participants whose sleep was obviously affected by in-home PSG were excluded from this study. However, we must note that the PSG effect on sleep, such as first night effect for PSG and sleep duration, cannot be avoided. Furthermore, single night PSG monitoring may not fully reflect sleep parameters. Multiple in-home PSGs over a long-period of time may provide additional details and decrease measurement error. Additionally, records regarding non-apnea sleep disorders such as restless legs' syndrome, hypersomnia, clinical insomnia, and REM behavior disorders were not included in SHHS datasets at baseline. Therefore we were unable to enroll this information in our analysis.

\section{Conclusion}

The current study found that longer WASO, poor SE, and short sleep duration were associated with the incidence of ischemic stroke. This significant difference still exists after the adjustment for potential risk factors. Sleep hygiene education, which could help people decrease sleep disruption, improve SE, and obtain suitable sleep duration, may be a helpful way to prevent the incidence of ischemic stroke.

\section{Highlights}

1. The relationship between objective sleep characteristics and the incidence ischemic stroke was investigated.

2. Increased WASO, low SE, and short sleep duration were associated with the incidence of ischemic stroke.

3. Objective sleep characteristics may be used as predictors to prevent the incidence of ischemic stroke.

\section{Acknowledgments}

We appreciate the Brigham and Women's Hospital for sharing the Datasets of Sleep Heart Health Study (SHHS). Moreover, SHHS acknowledges the Atherosclerosis Risk in Communities Study, the Cardiovascular Health Study, the Framingham Offspring and Omni Study, the Strong Heart Study, the Tucson Epidemiological Study of Obstructive Lung Disease, the cohort studies of respiratory disease in Tucson, and cohort studies of hypertension in New York. The Sleep Heart Health Study (SHHS) was supported by National Heart, Lung, and Blood Institute cooperative agreements U01HL53916 (University of
California, Davis), U01HL53931 (New York University), U01HL53934 (University of Minnesota), U01HL53937 and U01HL64360 (Johns Hopkins University), U01HL53938 (University of Arizona), U01HL53940 (University of Washington), U01HL53941 (Boston University), and U01HL63463 (Case Western Reserve University). The National Sleep Research Resource was supported by the National Heart, Lung, and Blood Institute (R24HL114473, 75N92019R002)." SHHS is particularly grateful to the members of these cohorts who agreed to participate in SHHS as well. SHHS further recognizes all the investigators and staff who have contributed to its success. A list of SHHS investigators, staff and their participating institutions is available on the SHHS website, https://doi.org/10.25822/ghy8-ks59.

\section{Author Contributions}

B.Y., and B.Z. raised the idea for the study. B.Y., B.Z., Y. W., X.J., L.Y., and J.Y. contributed to the study design, writing and review of the report. B.Y. and X.M. acquired the data in SHHS and B.Y. participated in further data analysis. B.Y. handled supervision in this study. All authors made a significant contribution to the work reported, whether that is in the conception, study design, execution, acquisition of data, analysis and interpretation, or in all these areas; took part in drafting, revising or critically reviewing the article; gave final approval of the version to be published; have agreed on the journal to which the article has been submitted; and agree to be accountable for all aspects of the work.

\section{Funding}

This study was supported by the Clinical Research Award of the First Affiliated Hospital of Xi'an Jiaotong University, China (No. XJTU1AF-CRF-2019-022).

\section{Disclosure}

The authors declare no conflicts of interest in this work.

\section{References}

1. Johnston SC, Mendis S, Mathers CD. Global variation in stroke burden and mortality: estimates from monitoring, surveillance, and modelling. Lancet Neurol. 2009;8(4):345-354. doi:10.1016/S14744422(09)70023-7

2. www.who.int/cardiovascular_diseases/resources/atlas/en/Xu W, Lin J, Gao M, et al. Rapid computer-aided diagnosis of stroke by serum metabolic fingerprint based multi-modal recognition. $A d v \quad S c i$ (Weinh). 2020;7(21):2002021. doi:10.1002/advs.202002021 
3. Bogiatzi C, Hackam DG, McLeod AI, Spence JD. Secular trends in ischemic stroke subtypes and stroke risk factors. Stroke. 2014;45 (11):3208-3213. doi:10.1161/STROKEAHA.114.006536

4. Zhou YP, Tian YF, Zhong CK, et al. Combined effects of family history of CVD and heart rate on ischemic stroke incidence among Inner Mongolians in China. Neurol Res. 2016;38(5):441-447. doi:10.1080/01616412.2016.1155816

5. Slomski A. Rapid blood pressure reduction safe for ischemic stroke. JAMA. 2019;321:1558.

6. Deijle IA, Van Schaik SM, Van Wegen EE, Weinstein HC, Kwakkel G, Van den Berg-vos RM. Lifestyle interventions to prevent cardiovascular events after stroke and transient ischemic attack: systematic review and meta-analysis. Stroke. 2017;48(1):174-179. doi:10.1161/STROKEAHA.116.013794

7. Zhang S, Chang C, Zhang J, Song B, Fang H, Xu Y. Correlation analysis of sleep quality and youth ischemic stroke. Behav Neurol. 2014;2014:246841. doi:10.1155/2014/246841

8. Ifergane G, Ovanyan A, Toledano R, et al. Obstructive sleep apnea in acute stroke: a role for systemic inflammation. Stroke. 2016;47 (5):1207-1212. doi:10.1161/STROKEAHA.115.011749

9. Zhou L, Yu K, Yang L, et al. Sleep duration, midday napping, and sleep quality and incident stroke: the Dongfeng-Tongji cohort. Neurology. 2020;94(4):e345-e356. doi:10.1212/WNL.00000000000008739

10. Gottlieb DJ, Punjabi NM, Newman AB, et al. Association of sleep time with diabetes mellitus and impaired glucose tolerance. Arch Intern Med. 2005;165(8):863-867. doi:10.1001/archinte.165.8.863

11. Doyle CY, Ruiz JM, Taylor DJ, et al. Associations between objective sleep and ambulatory blood pressure in a community sample. Psychosom Med. 2019;81(6):545-556. doi:10.1097 PSY.0000000000000711

12. Bowman MA, Duggan KA, Brindle RC, et al. Prospective associations among objectively and subjectively assessed sleep and the metabolic syndrome. Sleep Med. 2019;58:1-6. doi:10.1016/j. sleep.2019.02.005

13. Rosique-Esteban N, Papandreou C, Romaguera D, et al. Crosssectional associations of objectively-measured sleep characteristics with obesity and type 2 diabetes in the PREDIMED-plus trial. Sleep. 2018;41(12):zsy190. doi:10.1093/sleep/zsy190.

14. Massar SAA, Liu JCJ, Mohammad NB, Chee MWL. Poor habitual sleep efficiency is associated with increased cardiovascular and cortisol stress reactivity in men. Psychoneuroendocrinology. 2017;81:151-156. doi:10.1016/j.psyneuen.2017.04.013

15. Ramos AR, Weng J, Wallace DM, et al. Sleep patterns and hypertension using actigraphy in the Hispanic Community Health Study/Study of Latinos. Chest. 2018;153(1):87-93. doi:10.1016/j. chest.2017.09.028

16. Yan B, Yang J, Yang L, et al. Association of incident angina pectoris and rapid eye movement sleep in a large community-based study: the Sleep Heart Health Study. Sleep Med. 2019;59:7-14. doi:10.1016/j. sleep.2018.10.040

17. Javaheri S, Zhao YY, Punjabi NM, Quan SF, Gottlieb DJ, Redline S. Slow-wave sleep is associated with incident hypertension: the Sleep Heart Health Study. Sleep. 2018;41:zsx179.

18. von Kanel R, Loredo JS, Ancoli-Israel S, Mills PJ, Natarajan L, Dimsdale JE. Association between polysomnographic measures of disrupted sleep and prothrombotic factors. Chest. 2007;131 (3):733-739. doi:10.1378/chest.06-2006

19. Quan SF, Howard BV, Iber C, et al. The Sleep Heart Health Study: design, rationale, and methods. Sleep. 1997;20:1077-1085.

20. McMahon DM, Burch JB, Wirth MD, et al. Persistence of social jetlag and sleep disruption in healthy young adults. Chronobiol Int. 2018;35(3):312-328. doi:10.1080/07420528.2017.1405014

21. Wang C, Bangdiwala SI, Rangarajan S, et al. Association of estimated sleep duration and naps with mortality and cardiovascular events: a study of 116632 people from 21 countries. Eur Heart J. 2018;40(20):1620-9.
22. Shrivastava D, Jung S, Saadat M, Sirohi R, Crewson K. How to interpret the results of a sleep study. J Community Hosp Intern Med Perspect. 2014;4(5):24983. doi:10.3402/jchimp.v4.24983

23. Centers for Disease $\mathrm{C}$ and Prevention. Effect of short sleep duration on daily activities-United States, 2005-2008. MMWR Morb Mortal Wkly Rep. 2011;60:239-242.

24. Medic G, Wille M, Hemels ME. Short- and long-term health consequences of sleep disruption. Nat Sci Sleep. 2017;9:151-161. doi:10.2147/NSS.S134864

25. Van Den Berg JF, Van Rooij FJ, Vos H, et al. Disagreement between subjective and actigraphic measures of sleep duration in a population-based study of elderly persons. J Sleep Res. 2008;17 (3):295-302. doi:10.1111/j.1365-2869.2008.00638.x

26. Silva GE, Goodwin JL, Sherrill DL, et al. Relationship between reported and measured sleep times: the sleep heart health study (SHHS). J Clini Sleep Med. 2007;3:622-630.

27. Miner B, Stone KL, Zeitzer JM, et al. Self-reported and actigraphic short sleep duration in older adults. J Clini Sleep Med. 2021:jcsm9584.

28. Tsuchiyama K, Nagayama H, Kudo K, Kojima K, Yamada K. Discrepancy between subjective and objective sleep in patients with depression. Psychiatry Clin Neurosci. 2003;57(3):259-264. doi:10.1046/j.1440-1819.2003.01114.x

29. Lauderdale DS, Knutson KL, Yan LL, Liu K, Rathouz PJ. Self-reported and measured sleep duration: how similar are they? Epidemiology. 2008;19(6):838-845. doi:10.1097/EDE.0b013e318187a7b0

30. Girschik J, Fritschi L, Heyworth J, Waters F. Validation of self-reported sleep against actigraphy. $J$ Epidemiol. 2012;22 (5):462-468. doi:10.2188/jea.JE20120012

31. Gottlieb E, Landau E, Baxter H, Werden E, Howard ME, Brodtmann A. The bidirectional impact of sleep and circadian rhythm dysfunction in human ischaemic stroke: a systematic review. Sleep Med Rev. 2019;45:54-69. doi:10.1016/j.smrv.2019.03.003

32. Leng Y, Cappuccio FP, Wainwright NW, et al. Sleep duration and risk of fatal and nonfatal stroke: a Prospective Study and meta-analysis. Neurology. 2015;84(11):1072-1079. doi:10.1212/WNL.000 0000000001371

33. Zhang Y, Xie RP, Shen Y, Fan DS. Interaction between methylenetetrahydrofolate reductase C677T gene polymorphism and sleep duration on risk of stroke pathogenesis. Beijing Da Xие Xие Вао Yi Xие Ban. 2008;40:262-269.

34. Eguchi K, Hoshide S, Ishikawa S, Shimada K, Kario K. Short sleep duration is an independent predictor of stroke events in elderly hypertensive patients. J Am Soc Hypertens. 2010;4(5):255-262. doi:10.1016/j.jash.2010.09.001

35. Chen JC, Brunner RL, Ren H, et al. Sleep duration and risk of ischemic stroke in postmenopausal women. Stroke. 2008;39 (12):3185-3192. doi:10.1161/STROKEAHA.108.521773

36. Lutsey PL, McClelland RL, Duprez D, et al. Objectively measured sleep characteristics and prevalence of coronary artery calcification: the Multi-Ethnic Study of Atherosclerosis Sleep Study. Thorax. 2015;70(9):880-887. doi:10.1136/thoraxjnl-2015-206871

37. Campos-Rodriguez F, Martinez-Garcia MA, Reyes-Nunez N, CaballeroMartinez I, Catalan-Serra P, Almeida-Gonzalez CV. Role of sleep apnea and continuous positive airway pressure therapy in the incidence of stroke or coronary heart disease in women. Am J Respir Crit Care Med. 2014;189(12):1544-1550. doi:10.1164/rccm.201311-2012OC

38. Redline S, Yenokyan G, Gottlieb DJ, et al. Obstructive sleep apnea-hypopnea and incident stroke: the Sleep Heart Health Study. Am J Respir Crit Care Med. 2010;182(2):269-277. doi:10.1164/ recm.200911-1746OC

39. St-Onge MP, Grandner MA, Brown D, et al. Sleep duration and quality: impact on lifestyle behaviors and cardiometabolic health: a scientific statement from the American heart association. Circulation. 2016;134(18):e367-e386. doi:10.1161/CIR.00000 00000000444 
40. Castro-Diehl C, Diez Roux AV, Redline S, et al. Sleep duration and quality in relation to autonomic nervous system measures: the Multi-Ethnic Study of Atherosclerosis (MESA). Sleep. 2016;39 (11):1927-1940. doi:10.5665/sleep.6218
41. McAlpine CS, Kiss MG, Rattik S, et al. Sleep modulates haematopoiesis and protects against atherosclerosis. Nature. 2019;566 (7744):383-387. doi:10.1038/s41586-019-0948-2

\section{Publish your work in this journal}

Nature and Science of Sleep is an international, peer-reviewed, open access journal covering all aspects of sleep science and sleep medicine, including the neurophysiology and functions of sleep, the genetics of sleep, sleep and society, biological rhythms, dreaming, sleep disorders and therapy, and strategies to optimize healthy sleep.
The manuscript management system is completely online and includes a very quick and fair peer-review system, which is all easy to use. Visit http://www.dovepress.com/testimonials.php to read real quotes from published authors. 